\title{
Performance Analysis of Spiral Neighbourhood Topology Based Local Binary Patterns in Texture Recognition
}

\author{
Nihan Kazak*1, Mehmet Koc ${ }^{2}$
}

\author{
Accepted $3^{\text {rd }}$ September 2016
}

\begin{abstract}
In many texture recognition problems, Local Binary Patterns (LBP) method is used for feature extraction. This method is based on comparison of each centre pixel and its neighbours' intensity value in image. Due to its simplicity of calculation, LBP has become one of the most popular feature extraction techniques. In literature, different neighbourhood topologies of LBP structure are given such as circle, square, ellipse, parabola, hyperbola, and Archimedean spiral. This paper focuses on the use of uniform and basic LBP that have spiral topology in texture classification. We first derive basic and uniform LBP features based on spiral topology. Then the performances of several classification methods such as linear discriminant analysis (LDA), linear regression classifier (LRC), support vector machines (SVM), Chi-square test, and G-test are compared using these features in UIUC texture database.
\end{abstract}

Keywords: Local binary patterns, texture recognition, feature extraction, classification methods, spiral topology.

\section{Introduction}

Texture can be defined as a repeating pattern of local variations in image intensity. Texture contains information about the spatial arrangement of the colours or intensities in an image. Texture classification basically means that assignment of unknown image to one of known classes. Texture classification process consists of two main operations. The first operation is extracting features of the image and the second one is classification phase.

The best description of image is a very important step for classification phase of texture classification. There are a lot of feature extraction methods in literature for the best description of image. In [1], Raju and Durai presents texture classification techniques based on feature extraction in two categories according the local and the global features.

Local Binary Pattern (LBP) is one of the methods which used local features. It is gray-scale invariant local descriptor which was proposed by Ojala [2] and is a powerful descriptor for texture recognition. The descriptor is used in various fields of image processing problems such as shape localization [3], interest region description [4], gender classification [5], palm-print recognition [6], digital image stabilization [7] and especially face recognition problems [8]. There are a lot of improved versions of original LBP. In [9], authors present rotation invariant and uniform LBP which include fundamental information about texture. In [10], multi-dimensional LBP was proposed to using multi-dimensional histograms for different radii. Several versions of LBP were studied in [8].

The basic LBP calculates histograms with circular neighbourhood topology. Using this topology is advantage for rotation invariant problem in the texture classification application but anisotropic structural information may important features for some problems. An elliptical neighbourhood topology was used to exploit this anisotropic structural information for a face recognition system in

\footnotetext{
${ }^{1}$ Department of Computer Engineering, Bilecik Seyh Edebali University Bilecik/Turkey

${ }^{2}$ Department of Electrical and Electronics Engineering, Bilecik Seyh Edebali University Bilecik/Turkey

* Corresponding Author: Email: nihan.kazak@bilecik.edu.tr

Note: This paper has been presented at the $3^{\text {rd }}$ International Conference on Advanced Technology \& Sciences (ICAT'16) held in Konya (Turkey),
} September 01-03, 2016.
[11].

Other variants of LBP are proposed by [12]. Different shapes such as ellipse, circle, parabola, hyperbola, and Archimedean spiral for the neighbourhood calculation are tested. Different encodings to create LBP code are described for the evaluation of the local grayscale difference. According to this work, proposed quinary encoding and using elliptic neighbourhood performs the best with the medical image databases.

In the classification phase, performance of subspace based classification method is better than the statistical tests based on similarity measures [13]. We employed Chi-square test, G-test, support vector machines (SVM), linear discriminant analysis (LDA) and linear regression classifier (LRC) for the classification. LRC is one of the subspace methods [14] that is generally used for face recognition problems [15-17]. The experimental results of our study show that using LRC with SLBP features gives better results in texture recognition.

The remainder of the paper is organized as follows. Section 2 introduces the LBP operator. Our approach for computing the LBP codes is explained in Section 3 and classification methods are presented in Section 4. The experimental study performed in this paper is given in Section 5 and finally conclusions are presented in Section 6.

\section{LBP}

LBP proposed by Ojala et al. is one of the most famous texture descriptor. LBP operator is the grayscale invariant texture measure. This operator works with the gray value of image pixels.

For the each pixels of an image which is defined as centre pixel, binary code is generated by comparing it and its neighbours. If the difference between compared neighbouring pixel value and centre pixel value is higher or equal to 0 , compared neighbouring pixel value is coded as a binary 1 , in the other case it is coded 0 . Fig. 1 shows an example of LBP method.

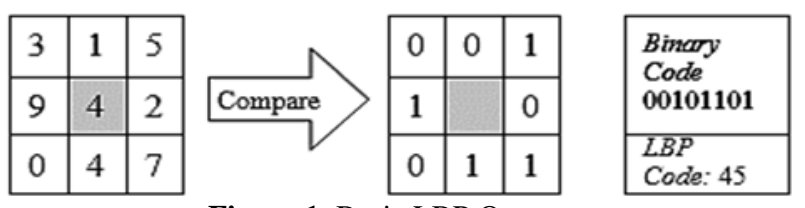

Figure 1. Basic LBP Operator 
The limitation of the basic LBP operator is using $3 \times 3$ square neighbourhoods for the description of local binary pattern. To eliminate the disadvantage, the operator was extended to use different size of neighbourhood and Fig. 2 illustrates two circularly symmetric neighbour sets for different radii.
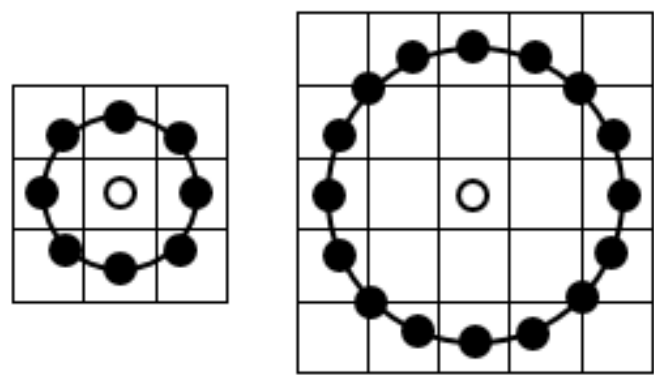

Figure 2. $(8,1)$ and $(16,2)$ LBP Operator

Let $L B P_{P, R}$ denote the LBP code of a pixel's circularly neighbourhoods, where $P$ is the number of sample points on the circle of radius $R, g_{c}$ represents intensity value of centre pixel and $g_{p}$ is the gray value of its $p$ th neighbour. Then the $L B P_{P, R}$ can be calculated as follows:

$$
L B P_{P, R}=\sum_{p=0}^{P-1} s\left(g_{p}-g_{c}\right) 2^{P}, s(x)= \begin{cases}1, & x \geq 0 \\ 0, & x<0\end{cases}
$$

As a result of calculation of LBP operator, decimal number is assigned to each pixel of image. After this process, a histogram is built to represent the image and used for pattern recognition as feature.

The concept of uniform patterns is to extract fewer feature of image texture. Any LBP pattern is called as uniform (ULBP) if the binary pattern consists of at most two bitwise transitions from 0 to 1 or vice versa. For example, binary code of the pattern that given in Fig. 1 is 00101101 . This pattern is not a uniform pattern because of having higher than two bitwise transitions. It has three 0-1 transitions and two 1-0 transitions. A pattern having 00011000 binary codes is uniform pattern. If each pixel in image has 8 neighbourhoods, there are totally 58 uniform patterns. In histogram, only one bin is allocated for the all non-uniform patterns so histogram consists of 59 bins.

\section{Classifiers}

\subsection{G-test}

G-test is defined as the following equation:

$$
G(S, M)=2 \sum_{b=1}^{B} S_{b} \log \frac{S_{b}}{M_{b}}
$$

where $B$ is the bins number of the histogram, $S_{b}$ and $M_{b}$ represents probabilities of sample and model distributions at bin b respectively. $S_{b} \log S_{b}$ is the same for each model during classification and there is no effect of constant scaling factor 2 on classification result so G-test can be simplified as:

$$
L(S, M)=\sum_{b=1}^{B} S_{b} \log M_{b}
$$

As a result, test sample $S$ is assigned to $M$ model which maximizes the L statistic.

\subsection{Chi-square $\left(\chi^{2}\right)$ Test}

Assume that we have two histograms $S$ and $M$ for comparison. $\chi^{2}$ test is computed as:

$$
d_{\chi^{2}}(S, M)=\sum_{i=1}^{B} \frac{\left(s_{i}-m_{i}\right)^{2}}{s_{i}+m_{i}}
$$

where $B$ is the number of bins, $s_{i}$ and $m_{i}$ represents value of $S$ and $M$ distributions at bin $i$, respectively. A simple nearestneighbour rule is used for classification.

\subsection{Support Vector Machine (SVM)}

The purpose of SVM is to select a hyperline or a hyperplane which separates the features of two classes. If features can be separated linearly, this procedure can be applied otherwise, features are mapped onto higher dimensional space that can separate the features linearly.

\subsection{Linear Discriminant Analysis (LDA)}

LDA is the most commonly used as dimensionality reduction technique and maximizes the linear separability of the groups belonging to different classes in data.

The aim of LDA is to maximize the distance between distributions of classes and to minimize variation in each class. Thus it can reduce dimensionality and save the information that is used in classification process at the same time. Objective function that is maximized in LDA defined as:

$$
J(\boldsymbol{w})=\frac{\boldsymbol{w}^{T} S_{B} \boldsymbol{w}}{\boldsymbol{w}^{T} S_{T} \boldsymbol{w}}
$$

where $S_{B}$ is between the classes scatter matrix, $S_{T}$ is within the class scatter matrix.

\subsection{Linear Regression Classification (LRC)}

Assume that we have $C$ classes with $N$ training images. The matrix $W_{i}$ contains all feature vectors from $i$ th class. Thus

$$
W_{i}=\left[x_{i}^{1}: \cdots \vdots x_{i}^{j}: \cdots \vdots x_{i}^{N}\right]
$$

where $x_{i}^{j}$ represents the $j$ th feature vector of the $i$ th class. If $y$ belongs to the $i$ th class, it should be represented as a linear combination of the training images from the same class and can be defined as

$$
y=W_{i} \beta_{i}+\varepsilon
$$

where $\beta_{i}$ is the vector of parameters that is estimated using least squares estimation. Hence the estimation of $\beta_{i}$ is

$$
\widehat{\beta}_{\imath}=\left(W_{i}^{T} W_{i}\right)^{-1} W_{i}^{T} y
$$

The estimation of the vector $y$ is calculated as follow:

$$
\widehat{y}_{l}=W_{i}\left(W_{i}^{T} W_{i}\right)^{-1} W_{i}^{T} y
$$

The minimum distance between the original vector and the projected vector is calculated according to the Euclidean distance measure, i.e.,

$$
\operatorname{argmin}_{i}\left\{\left\|y-\hat{y}_{i}\right\|\right\}, \quad i=1,2, \ldots, C .
$$

\section{Spiral Local Binary Pattern (SLBP)}

We introduced basic encoding LBP operator with spiral topology for texture recognition in this section. The basic LBP calculates histograms with circular neighbourhood topology. Using this topology provides rotation invariant but cannot exploit the anisotropic structural information. This information may an important source in some problems. An elliptical neighbourhood topology has been used to exploit this anisotropic structural information for a face recognition system in [11].

Other variants of LBP are proposed by [12]. Different shapes such as ellipse, circle, parabola, hyperbola and Archimedean spiral for the neighbourhood calculation have been tested and different encodings have been used for the evaluation of the local grayscale difference. According to this work, proposed quinary encoding and using elliptic neighbourhood gives the best accuracy with the medical image databases. 
The equation of the Archimedean spiral is expressed in terms of polar coordinates such as $(r, \theta)$. The equation of Archimedean spiral can be described as follows

$$
r=a+b \theta
$$

where $a$ and $b$ can be any real numbers. Changing the parameter $a$ turns the spiral, while $b$ controls the distance between the turnings. Fig. 3 illustrates an example of spiral neighbour which we used to extract features.

Each pixel in the image is selected as the centre point respectively. Black circle is the centre pixel and its eight neighbours are represented as blue stars in Fig. 3.

The neighbour points are identified by choosing theta intervals of $\pi / 4$. If a point doesn't overlap to the centre of the pixel, the intensity value of the point is interpolated by bilinear interpolation.

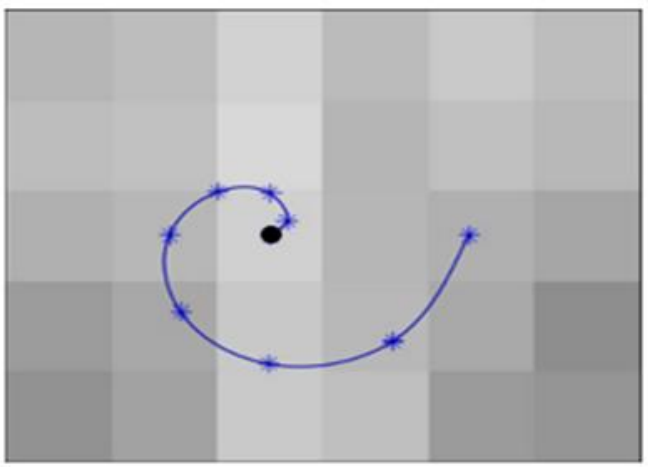

Figure 3. Spiral neighborhood topology

Calculation of LBP code is the same as basic LBP for SLBP. Firstly the nearest neighbour of centre pixel is compared and then comparison continues towards the farthest one. Consequently binary code is generated depending on whether the central pixel is higher than or equal to neighbours. Obtained binary code is converted to decimal value and this value is labelled to centre pixel. Finally histogram of decimal values is created.

Uniform spiral LBP (USLBP) is determined like uniform LBP and non-uniform ones correspond to only one bin in histogram.

\section{Experimental Results}

This section describes experiments that show the efficiency of the proposed spiral neighbourhood topology for texture classification.

UIUC texture database which involves 40 textures from each of 25 textures is used for evaluation. Fig. 4 shows example images from each class (the resolution of the samples is $640 \times 480$ pixels). Samples of each class have different viewpoint and scale differences. Furthermore, the dataset includes non-rigid deformations, illumination changes and viewpoint-dependent appearance variations.

To get statistically significant experimental results, 30 training images are randomly selected from each class of UIUC database while the remaining images per class are used as the validation set.

The Archimedean spiral starts in a centre pixel and makes a curve with one round $(2 \pi)$. So 8 sampling points on a spiral are computed intervals of $\pi / 4$. Sampling points which do not overlap to centre of a pixel are computed by bilinear interpolation. In (11), $b$ parameter is set as 0.3 for the best performance of spiral neighbourhood topology.

All texture images of UIUC database are represented by LBP, ULBP, SLBP and SULBP histograms. The LBP codes are computed using 8 sampling points on a circle of 1 radius for comparison with SLBP.

The extracted features are classified with Linear SVM, LDA, LRC, Chi-square test and G-test for measure of dissimilarity between features.

The classification algorithms are implemented ten times independently. The average classification accuracies obtained with different texture databases are shown in Table I. The best recognition result achieved is $85.64 \%$ with LRC where the SLBP features of UIUC database is selected as shown in Table I as bold and underlined style.

Performance of proposed SLBP is higher than the other feature extraction methods for all classification methods. The statistical tests based on similarity measures perform worse from subspace based methods. LRC is the best classification method in UIUC texture database as a result of experiments.
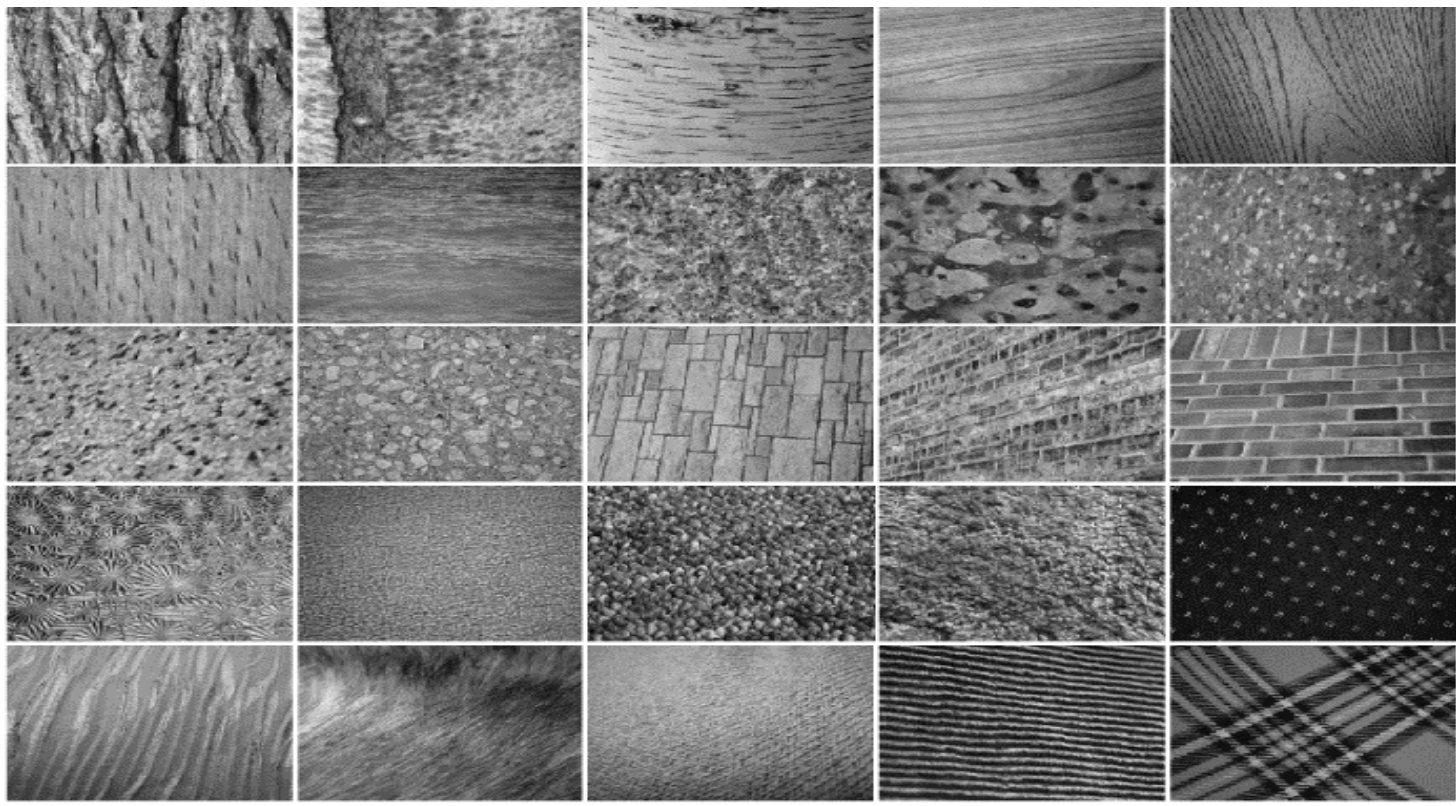

Figure 4. Sample images from UIUC texture dataset 
Table 1. Classification accuracies (\%)

\begin{tabular}{lllll}
\hline & LBP & ULBP & SLBP & SULBP \\
\hline \multirow{2}{*}{ G-test } & $\% 33.40$ & $\% 33.00$ & $\% 33.72$ & $\% 31.04$ \\
& \pm 1.98 & \pm 1.92 & \pm 2.73 & \pm 3.00 \\
\multirow{2}{*}{ Chi-square } & $\% 63.24$ & $\% 61.76$ & $\% 63.88$ & $\% 60.76$ \\
& \pm 2.35 & \pm 3.01 & \pm 2.81 & \pm 2.40 \\
\multirow{2}{*}{ SVM } & $\% 63.24$ & $\% 74.2$ & $\% 80.84$ & $\% 69.52$ \\
& \pm 2.35 & \pm 3.87 & \pm 2.55 & \pm 3.59 \\
\multirow{2}{*}{ LDA } & $\% 79.96$ & $\% 80.16$ & $\% 83.32$ & $\% 83.72$ \\
& \pm 3.50 & \pm 3.20 & \pm 2.60 & \pm 3.04 \\
\multirow{2}{*}{ LRC } & $\mathbf{\% 8 4 . 4 4}$ & $\mathbf{\% 8 3 . 2 8}$ & $\mathbf{\% 8 5 . 6 4}$ & $\mathbf{\% 8 4 . 3 6}$ \\
& \pm 2.34 & \pm 1.45 & \pm 1.91 & \pm 2.19 \\
\hline
\end{tabular}

\section{Conclusions}

In this paper, we have presented uniform and basic Local Binary Pattern (LBP) with Archimedean spiral neighbourhood topology to texture recognition. Firstly, we derived basic and uniform LBP features based on spiral topology. Then the performances of several classification methods such as linear discriminant analysis (LDA), linear regression classifier (LRC), support vector machines (SVM), Chi-square test, and G-test are compared using these features.

Experimental results obtained from UIUC texture database clearly demonstrate that the best recognition rate is obtained with using SLBP features and LRC method. In the future, we plan to focus on improving spiral topology to obtain more meaningful information.

\section{References}

[1] J. Raju ve C. Durai, A Survey on Texture Classification Techniques, Information Communication and Embedded Systems (ICICES), Chennai, 2013.

[2] T. Ojala, M. Pietikainen ve D. Harwood, A Comparative Study of Texture Measures with Classification Based on Feature Distributions, Pattern Recognition, vol. 29, no. 1, pp. 51-59, 1996.

[3] X. Huang, S. Li ve Y. Wang, Shape localization based on statistical method using extended local binary pattern, Proc.Int.Conf.Image Graph., 2004.

[4] M. Heikkila, M. Pietikainen ve C. Schmid, Decription of interest regions with local binary patterns, Pattern Recognition, vol. 42, pp. 425-436, 2009.

[5] S. C., Learning local binary patterns for gender classification on real-world face images, Pattern Recognition Letters, vol. 33, pp. 431-437, 2012.

[6] M. G. K. Ong, T. Connie ve T. A. B. Jin, Touch-less palm print biometrics: Novel design and implementation, Image and Vision Computing, vol. 26, pp. 1551-1560, 2008.

[7] B. Kir, M. Kurt ve O. Urhan, Local Binary Pattern Based Fast Digital Image Stabilization, IEEE Signal Processing Letters, vol. 22, pp. 341-345, 2015.

[8] S.-M. Huang ve J.-F. Yang, Linear Discriminant Regression Classification for Face Recognition, IEEE Signal Processing Letters, vol. 20, no. 1, pp. 91-94, 2013.

[9] T. Ojala, M. Pietikainen ve T. Maenpaa, Multiresolution Gray-Scale and Rotation Invariant Texture Classification with Local Binary Patterns, IEEE Trans. Pattern Analysis and Machine Intelligence, vol. 24, no. 7, pp. 971-987, 2002.

[10] G. Schaefer ve N. P. Doshi, Multi-dimensional Local Binary Pattern Descriptors for Improved Texture Analysis, 21st International Conference on Pattern Recognition (ICPR 2012), Tsukuba, Japan, 2012.

[11] S. Liao ve A. Chung, Face recognition by using enlongated local binary patterns with average maximum distance gradient magnitude, Computer Vision - ACCV 2007. Lecture Notes in Computer Science, vol. 4844, Berlin, Springer, 2007, p. 672-679.

[12] L. Nanni, A. Lumini ve S. Brahnam, Local binary patterns variants as texture descriptors for medical image analysis, Artificial Intelligence in Medicine, vol. 49, pp. 117-125, 2010.

[13] N. Kazak, M. Koc, B. Benligiray ve C. Topal, A comparison of classification methods for local binary patterns, 24th Signal Processing and Communication Application Conference (SIU), Zonguldak, 2016.

[14] I. Naseem, R. Togneri ve M. Bennamoun, Linear Regression for Face Recognition, IEEE Trans. Pattern Analysis and Machine Intelligence, pp. 2106-2112, 2010.

[15] I. Naseem, R. Togneri ve M. Bennamoun, Robust regression for face recognition, Pattern Recognition, vol. 45, pp. 104$118,2012$.

[16] M. Koc ve A. Barkana, Application of Linear Regression Classification to Low Dimensional Datasets, Neurocomputing, vol. 131, pp. 331-335, 2014.

[17] A. Suruliandi, Local binary pattern and its derivatives for face recognition, Computer Vision, IET, vol. 6, pp. 480488 ,

2012 